\title{
Unilateral neonatal glaucoma (buphthalmos) with persistent hyperplastic primary vitreous
}

\author{
Shashi Kumar Bhasker, Astha Jain, P.K. Srivastava \\ Department of Ophthalmology, King George's Medical University - UP, Lucknow, \\ India
}

\begin{abstract}
Leukocoria or white pupillary reflex in infancy or childhood is mostly associated with retinoblastoma (an intraocular malignancy). The possibility is increased if buphthalmos is also present, because non-malignant conditions like persistent hyperplastic primary vitreous (PHPV) usually have normal or small eyes. They rarely present with enlarged buphthalmic eye. Such was the condition in our neonate who presented with buphthalmos and leukocoria and was referred to us for management having been diagnosed with retinoblastoma. However, on investigating by imaging techniques (ultrasonography, Doppler and CT Scan) PHPV was confirmed, which is non-malignant. The patient was managed accordingly without enucleation. Even though rare, neonates or infants presenting with buphthalmos and leukocoria, should be investigated for non-malignant conditions like PHPV by imaging techniques to rule out a malignant condition - retinoblastoma.
\end{abstract}

Key words: Leukocoria, persistent hyperplasic primary vitreous (PHPV), buphthalmos

\section{Introduction}

Persistent hypertrophic primary vitreous (PHPV) is a congenital condition where remnants of fetal vasculature stay behind after birth. The eye in this condition is usually microphthalmic and may be associated with white pupillary reflex (leukocoria) and poor vision. It is rare to have buphthalmic eye in a patient with PHPV, presenting with white pupillary reflex. To the best of our knowledge only Wallace Alward et al. reported a case of PHPV who presented as buphthalmos at birth in both eyes. ${ }^{1}$ Two cases of PHPV presenting as buphthalmos have also been reported by Ming-Hui Sun et al. ${ }^{2}$ and Khan. ${ }^{3}$ Both cases were of more than five weeks of age. We present a rare case of PHPV (both anterior and posterior type) who presented as buphthalmos since birth.

\section{Case report}

A 15-days-old male neonate was presented by his parents in the outpatient department of ophthalmology of a tertiary care institute, with complaints of yellowish white discoloration of the left eye since birth (Fig. 1). The discoloration in the left eye was first noticed by the mother. It was not associated with excessive crying, redness or watering. The parents took the baby to a district hospital, from where he was referred to our tertiary care hospital for further management, with the

Correspondence: Dr. Shashi Kumar Bhasker, Associate Professor, Glaucoma Services, Department of Ophthalmology, King George's Medical University - UP, Lucknow - 226003, INDIA. E-mail: skbhasker@yahoo.com 
diagnosis of retinoblastoma.

Birth of neonate was at full term with a normal vaginal delivery. He was normal and cried actively after birth. The APGAR score was 10 and birth weight was 2600 grams.

Antenatal history was not significant and no consanguinity was present. He was the second child after a girl who was normal.

On examination, the neonate was healthy and active. The general and systemic examination did not reveal any abnormality. Ocular examination showed normal lids and conjunctiva in both eyes. The patient closed his right eye when light was shown in it but there was no response in the left eye.

In the right eye, the cornea was clear and measured eight $\mathrm{mm}$ in both the horizontal and vertical axis. Anterior chamber was normal with normal pupil. Fundus seen through clear lens was normal. Direct pupillary reaction was present with no consensual reaction in right eye. The intraocular pressure was $10 \mathrm{mmHg}$

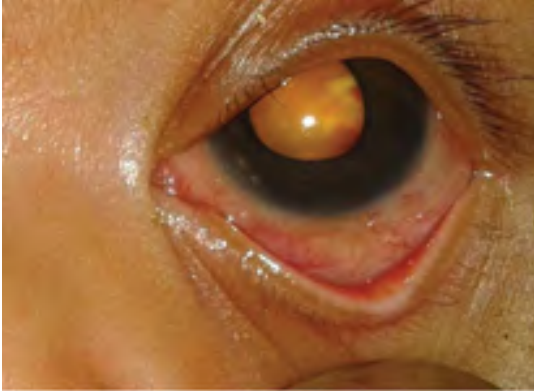

Fig. 1. Clinical photograph of neonate showing leukocoria with a few hemorrhagic spots and enlarged globe. (measured by rebound tonometer). On gonioscopic examination by Koeppe's goniolens, the angle structures could be discerned. This confirmed that the angle was open in the right eye.

In the left eye, a mild diffuse haze was seen in the cornea. Corneal diameters were $12 \mathrm{~mm}$ in both horizontal and vertical axis. Anterior chamber was shallow. Ectropion uveae with neovascularization was seen all around. The pupillary reflex was yellowish white with a few hemorrhagic red spots (intralenticular)(Fig. 1). Both direct and consensual pupillary reflexes were absent.

Intraocular pressure was $20 \mathrm{mmHg}$ (measured by rebound tonometer). Angle structures could not be identified on direct gonioscopic evaluation by Koeppe's goniolens due to peripheral iridocorneal contact and haziness of cornea. Also, the angle in this left eye was markedly narrow or, in other words, it was closed (all round $360^{\circ}$.

\section{Investigations}

The hemogram showed normal blood counts and hemoglobin for that age.

Ultrasonography of left orbit showed enlarged size of the globe $(17.2 \mathrm{~mm}$ in the anteroposterior axis and $17.9 \mathrm{~mm}$ in the horizontal axis). Anterior segment shows thinning of lens with shallow anterior

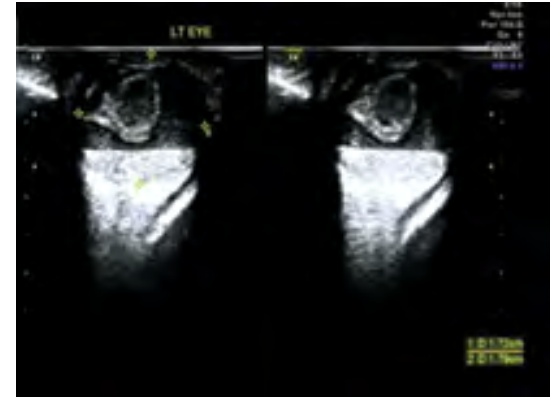

Fig. 2. High-resolution ultrasonography of the patient showing membranes and fluid levels in the posterior segment. Membranes also include detached retina. 
chamber. Vitreous membranes could be visualized in the vitreous cavity. A persistent retrolenticular hyaloid vasculature is seen through the vitreous cavity to the posterior pole. Layered fluid level is present in the posterior part of the globe along with hyaloid and retinal detachment (Fig. 2).

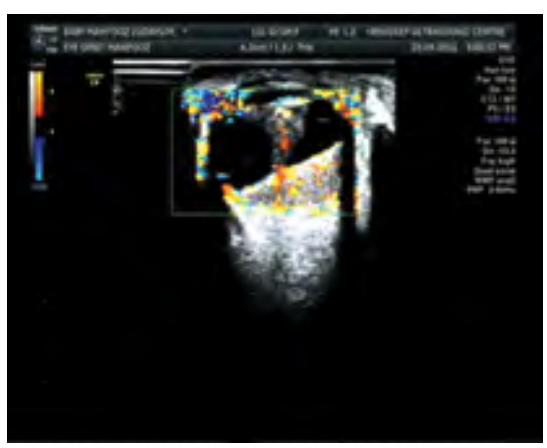

Fig. 3. Colored Doppler ultrasonography showing a retrolenticular vasculature extending from the lens to the optic disc. Hemorrhagic fluid levels are also visualized.

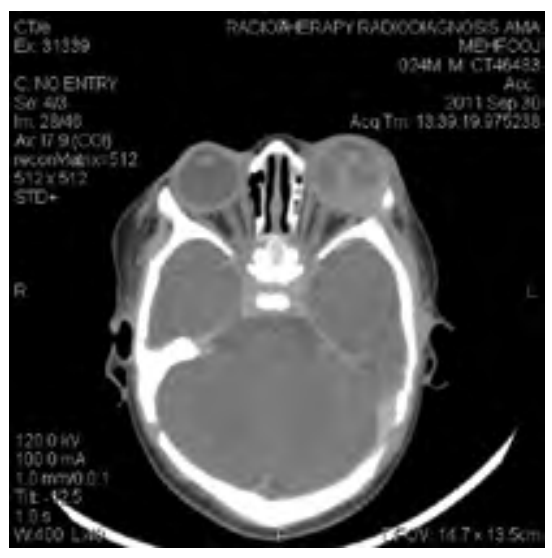

Fig. 4. Axial CT scan of the left orbit showing radio opaque shadow hyaloids vasculature from the lens to the optic disc. Retinal detachment can be seen. Fluid level at the posterior pole present due to hemorrhage from hyaloid vessels. Dysgenesis of angle structures is also seen.
Color Doppler ultrasonography shows a retrolenticular vascular structure extending from the posterior pole to the back of the posterior capsule of the lens - a sign of persistent hyaloid vasculature. Anteriorly, remnants of the posterior part tunica vasculosa lentis, extending into the posterior capsule of the lens (along with intralenticular hemorrhage), can be visualized. The anterior tunica vasculosa lentis could be appreciated in the periphery. Vascularized membranes extending to the anterior chamber angles are also present. So the anterior chamber angle deformity is expected to lead to failure in draining of aqueous. In the posterior part of the globe, layered fluid (hemorrhage) levels are seen.

Axial CT Scan of the left orbit shows the same features as shown by B scan ultrasonography and Doppler sonography. The anterior chamber is shallow. Presence of hyaloid vasculature along with retinal and hyaloid detachment is seen. Anteriorly, the tunica vasculosa lentis can be seen invading the posterior capsule of lens and the angle of anterior chamber. The lens is thin to membranous in shape and a radiopaque shadow which may be due to intra lenticular hemorrhage is present. Layered fluid collection is visualized at the posterior pole. The globe of the left eye is larger than that of the right eye. Optic nerve as seen on the scan is thinner than in the right eye (perhaps due to hypotrophy or atrophy) (Fig. 4).

The right eye was normal on the CT scan.

The parents were informed about the visual status of their baby and trabeculectomy followed by vitrectomy in the left eye was recommended. They refused surgery as this would not lead to any visual gain. However, cryoapplication was done in the inferior half at the limbus. After one week, the cornea cleared and the 
IOP was $14 \mathrm{mmHg}$ on rebound tonometer. The patient's parents were advised to bring the baby after one month. As they failed to do so, the patient could not be followed up.

\section{Discussion}

During the development of the eye, the optic pit evaginates from the prosencephalon (forebrain) on day 23 leading to formation of the optic vesicle on day 25 (3-mm stage). Eccentric invagination of the optic vesicle occurs to form the optic cup with embryonic fissure on day 27 (5-7-mm stage).

The hyaloid artery, along with mesenchyme, gains access into the optic cup through the embryonic fissure. The embryonic fissure closes at day 33 (13-13-mm stage). The hyaloid artery forms a vascular network in the optic cup and at the posterior lenticular surface as the posterior vasculosa lentis. All this forms the primary vitreous, which is well formed by week 9 . From month 3-4, atrophic changes start and this primary vitreous is completely absorbed by month 7 and disappears by the time of birth. The absorption starts in the central portion and extends both anteriorly and posteriorly. Secondary vitreous replaces the primary.

Simultaneously, the invaginating optic cup induces the formation of the lens from the surface ectoderm through the lens vesicle. The lens vesicle separates from the surface ectoderm and moves inside the optic cup. Later the surface ectoderm and the mesenchyme surrounding the anterior portion of the optic cup forms the cornea, anterior chamber, angle structures iris stroma and ciliary body.

Persistence along with hypertrophy of the hyaloid vasculature (fetal vasculature) leads to a condition known as persistent hypertrophic primary vitreous (PHPV). This PHPV may be purely anterior, purely posterior or a combination of both. The anterior type is also known as persistent tunica vasculosa lentis or persistent posterior fetal fibrovascular sheath of the lens. The posterior variety is also known as falciform retinal septum or ablatio falciformis congenita. Other ocular abnormalities or systemic malformations may be associated with PHPV. ${ }^{4}$

Persistent hypertrophic primary vitreous may be bilateral $(10 \%)$ or unilateral (90\%). Patient usually presents with severe visual loss, in the early stages leukocoria and in later stages phthisical eye or glaucomatous eye. The eye is either normal or microphthalmic and may be associated with white pupillary reflex (leukocoria).

Alward et al. report a case of bilateral buphthalmos in a neonate of four days. ${ }^{1} \mathrm{He}$ had an anterior and posterior variety of PHPV. On fundus examination, the hyaloid vasculature was traceable to the optic disc. The cup-to-disc ratio was 0.8 in both eyes. After management by trabeculotomy, lensectomy and vitrectomy, the child could fix and follow with both eyes. So this case had some normal retinal tissue which helped him in some visual gain.

Our case also had congenital glaucoma (buphthalmos) with PHPV (both anterior and posterior) in one eye only (left). But the malformation in the anterior and posterior segment was more severe, as evidenced by the persistence of blood flow in the retrolenticular hyaloid vasculature, intralenticular and posterior segment hemorrhage, along with retinal detachment and angle dysgenesis. Recurrent hemorrhage 
in the posterior segment and in the lens along with rubeosis and angle malformation/dysgenesis is the pathophysiology for increase in globe size (buphthalmos) and increase in intraocular pressure. Any functional visual gain by surgery (vitrectomy, lensectomy and/or trabeculectomy) was not possible as it was in the case reported by Alward et al., ${ }^{1}$ the reason being that the optical and neuroretinal parts of the visual axis were showing malformation and dysgenesis associated with retinal detachment and hemorrhage. As no functional retina was present, there was little chance of any visual gain. In addition to this, the parents did not consent for any surgical intervention.

To our knowledge, this case is the first of its kind reported in this part of the world, and it may be the first worldwide. Genetic study would have helped us understand the molecular basis for buphthalmos associated with PHPV at birth (two comorbidities existing together), but we do not have this facility. Moreover, it would have unraveled the mystery of the condition occurring unilaterally and that too in a more severe form. Had any genetic mutation taken place, it should have occurred bilaterally as in the case reported by Alward et al. ${ }^{1}$

\section{Conclusion}

Though rare, patients with buphthalmos and/or leukocoria should be investigated by imaging techniques for PHPV and other non-malignant conditions, to rule out retinoblastoma, which is a malignant condition. This will prevent unnecessary enucleation.

\section{References}

1. Alward WLM, Krasnow MA, Keech RV, Pulido JS, Sutton GL. Persistent hyperplastic primary vitreous with glaucoma presenting in infancy. Arch Ophthalmol 1991;109:1063-1064.

2. Sun MH, Kao LY, Kuo YH. Persistent hyperplastic primary vitreous: Magnetic resonance imaging and clinical findings. Chang Gung Med J 2003;26:269-276.

3. Khan AO. Buphthalmos in the setting of persistent hyperplastic primary vitreous cataract. Am J Ophthalmol 2003;136:945-947.

4. Haddad R, Font RL, Reeser F. Persistent hyperplastic primary vitreous. A clinicopathological study of 62 cases and review of the literature. Surv Ophthalmol 1978;23(2):123-134. 